\title{
Role of caking in optimizing the performance of a concertinaed ceramic filtration membrane
}

\author{
V. E. Pereira ๑, M. P. Dalwadi ๑, and I. M. Griffiths $\odot^{*}$ \\ OCIAM, Mathematical Institute, University of Oxford, Oxford OX2 6GG, United Kingdom
}

(Received 12 May 2021; accepted 31 August 2021; published 1 October 2021)

\begin{abstract}
Membrane filtration is a process of separating particles from fluids. Over time, particles are trapped within the membrane structure and on the membrane surface, forming a cake. In this paper, we develop a mathematical model for the transient blocking dynamics in a concertinaed filtration device composed of angled porous membranes and dead-ends. We examine how the inclusion of particles affects the flow dynamics, and we uncover potential inaccuracies in relying on flux-throughput curves to distinguish between caking and internal blocking dynamics. Moreover, we show that optimal filtration performance strongly depends on both the performance metric and the membrane configuration. Finally, to optimize the use of membrane area, we introduce a method for deriving a nonuniform permeance that ensures constant initial cake growth.
\end{abstract}

DOI: 10.1103/PhysRevFluids.6.104301

\section{INTRODUCTION}

Membrane filtration is a vital process used to separate fluid mixtures in many industry sectors including healthcare, water treatment, and food production [1-3]. Filtration devices comprise porous membranes through which a mixture of fluid and unwanted particles is passed. The particles get trapped by the membrane and a filtered fluid is produced. While efficient instantaneous trapping of unwanted particles makes for a good filter, this occurs at the cost of blocking the filter over time, which can reduce the total amount of fluid processed by the device. There are two key blocking mechanisms: blocking inside the membrane pores (internal blocking) and blocking on the surface of the membrane (caking).

There are a wide range of filtration devices and in this paper we focus our attention to direct-flow filtration devices. Such devices consist of stacked porous membranes with alternating capped ends [see Fig. 1(a)]. The fluid mixture is passed into the device through open ends, travels through the porous membrane and then out through the other side through open ends. Typically, in direct-flow devices, the fluid is fed in parallel to the membrane surface. In this paper, we seek to understand how filtration performance is affected by an angled-membrane configuration in a direct-flow device. The motivation for this study arises from an industrial example of such a direct-flow device designed by Smart Separations Ltd [4]. The steady-state version of the model presented herein is derived in earlier work [5]. The goal of the previous study was to optimize the filtration performance for a particle-free steady flow. In this paper we build on this previous work to incorporate transient blocking effects in the optimization of the performance of a concertinaed filtration system.

An understanding of the blocking dynamics in filtration is vital in optimizing the design of a filter, and there have been a number of previous studies examining such dynamics. In [6], the authors developed a mathematical model of internal blocking in vertically stacked filtration membranes in a direct-flow device. This previous study was concerned with the effect of spacing between

*ian.griffiths@maths.ox.ac.uk 


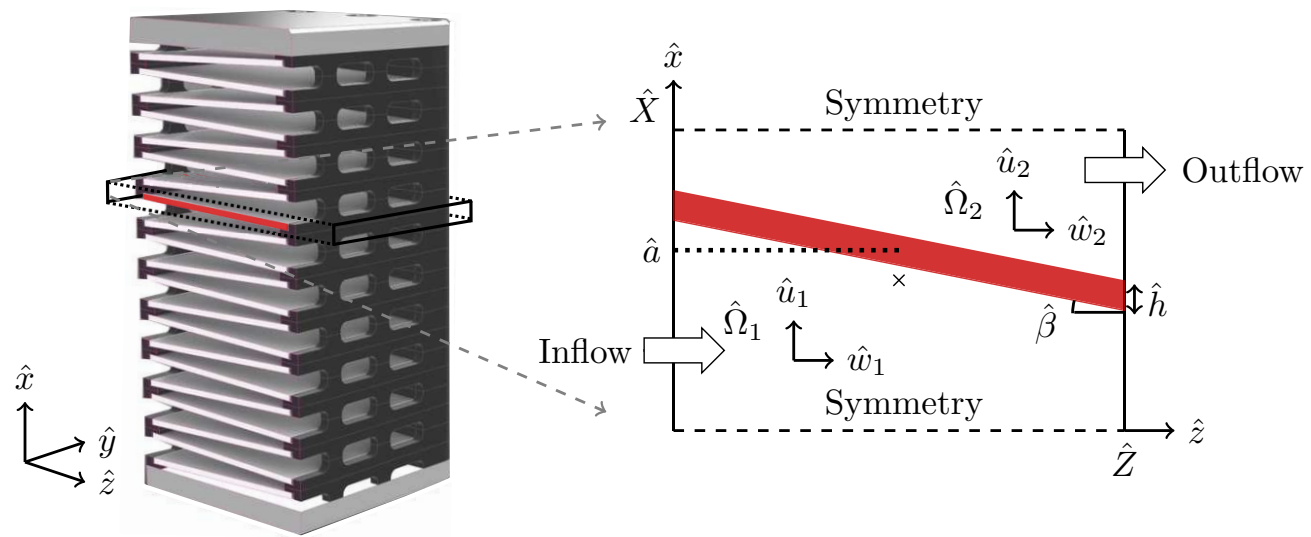

(a)

(b)

FIG. 1. Schematic of the concertinaed filtration device. (a) The full three-dimensional device; reproduced from Ref. [4] with permission. (b) The two-dimensional domain of a single filtration module; the center of the membrane is $(\hat{x}, \hat{z})=(\hat{a}, \hat{Z} / 2)$ and the center of the domain $(\hat{x}, \hat{z})=(\hat{X} / 2, \hat{Z} / 2)$ is indicated by a cross.

the membranes on the total amount of fluid processed through the device. Further relevant work arises from studies of blocking in pleated filtration membranes, which are geometrically similar to a direct-flow device with the key difference being the presence of dead-ends in the latter. In Ref. [7], the authors presented an asymptotic analysis of caking dynamics relevant for several different parameter regimes applied to a rectangularly pleated device. In Ref. [8], the authors incorporated both caking and internal-blocking dynamics in a mathematical model of flow through a rectangular pleated membrane with surrounding porous support material. The work sought to examine the relative importance of the resistance of the support material to that of the membrane and the evolution of the permeability profile through internal blocking. Recently in Ref. [9], the authors presented a model of internal blocking within an analogous device of rectangular pleats contained in porous support material. The authors compared linear, quadratic, and cubic membrane permeability profiles with the aim of maximizing the total amount of fluid processed through the filtration device.

In this paper we present a mathematical model of caking dynamics in a direct-flow device with angled membranes. The distinction between the past works of blocking dynamics in a filtration device and the work presented in this paper is that we focus our study on the effects of particle inclusion, membrane configuration (namely angle and position), and membrane permeability profile on the caking dynamics in a concertinaed filtration device.

\section{MODEL DEVELOPMENT}

In this paper, we consider the flow through a single repeated module of the filtration device shown in Fig. 1. While there is design scope for the geometric parameters and we give a range as such in Table I, the geometry of the device is always such that the transverse length $\hat{X}$ is significantly smaller than the lateral length $\hat{Z}$ and depth $\hat{Y}$. Moreover, variations in the $\hat{y}$ axis are assumed to be negligible, and we consider the two-dimensional domain in $(\hat{x}, \hat{z})$ space [see Fig. 1(b)]. We will derive the model using dimensional quantities (denoted by hats) and then scale the model to derive the associated dimensionless quantities (denoted without hats). The position of the upstream side of the membrane is specified by $\hat{x}=\hat{m}(\hat{z})$ with

$$
\hat{m}(\hat{z})=\hat{a}+\frac{1}{2}(\hat{Z} \tan \hat{\beta}-\hat{h})-\hat{z} \tan \hat{\beta},
$$


TABLE I. Parameter values for air flow through the filtration device shown in Fig. 1; values for $\hat{Z}, \hat{Y}, \hat{X}, \hat{h}$, and $\hat{W}_{0}$ provided in Ref. [4].

\begin{tabular}{ll}
\hline \hline Parameter & Value \\
\hline Lateral height, $\hat{Z}$ & $50-60 \mathrm{~mm}$ \\
Filter depth, $\hat{Y}$ & $50-60 \mathrm{~mm}$ \\
Transverse length, $\hat{X}$ & $1-3.6 \mathrm{~mm}$ \\
Membrane thickness, $\hat{h}$ & $0.3-1.2 \mathrm{~mm}$ \\
Air viscosity, $\hat{\mu}$ & $1.81 \times 10^{-5} \mathrm{~Pa} \mathrm{~s}$ \\
Air density, $\hat{\rho}$ & $1.2 \mathrm{~kg} \mathrm{~m}^{-3}$ \\
Lateral velocity scale, $\hat{W}_{0}$ & $50 \mathrm{~mm} \mathrm{~s}^{-1}$ \\
\hline \hline
\end{tabular}

where $\hat{\beta}$ is the angle of the membrane with the horizontal axis and $\hat{h}$ is the membrane thickness [see Fig. 1(b)]. The membrane length $\hat{L}=\hat{Z} / \cos \hat{\beta}$ is governed by the angle $\hat{\beta}$. The position of the membrane midpoint $(\hat{x}, \hat{z})=(\hat{a}, \hat{Z} / 2)$ is determined through the parameter $\hat{a}$. The membrane separates the flow domain into two subdomains described by

$$
\begin{aligned}
& \hat{\Omega}_{1}=\{\hat{x} \in[0, \hat{s}(\hat{z}, \hat{t})], \hat{z} \in[0, \hat{Z}]\}, \\
& \hat{\Omega}_{2}=\{\hat{x} \in[\hat{m}(\hat{z})+\hat{h}, \hat{X}], \hat{z} \in[0, \hat{Z}]\},
\end{aligned}
$$

where $\hat{x}=\hat{s}(\hat{z}, \hat{t})$ is the position of the moving cake front at time $\hat{t}$.

Driven by a pressure drop $\left(\hat{p}_{\text {in }}-\hat{p}_{\text {out }}\right)>0$, where $\hat{p}_{\text {in }}$ and $\hat{p}_{\text {out }}$ are the inlet and outlet pressures, respectively, the flow enters the domain $\hat{\Omega}_{1}$ at $\hat{z}=0$, travels into the cake at $\hat{x}=\hat{s}(\hat{z}, \hat{t})$, through to the membrane at $\hat{x}=\hat{m}(\hat{z})$, and exits the domain $\hat{\Omega}_{2}$ at $\hat{z}=\hat{Z}$. The fluid mixture comprises fluid and particles, and the particles are filtered out by the membrane in $\hat{\Omega}_{1}$ resulting in a purified fluid in $\hat{\Omega}_{2}$.

The steady-state flow studied in our previous paper [5] is reversible and the results for $\hat{\beta}>0$ can be generalized to those for $\hat{\beta}<0$ with the subdomains $\hat{\Omega}_{1}$ and $\hat{\Omega}_{2}$ switched for symmetric flux. However, in this paper we consider the transient system with an evolving cake front in which the flow is not reversible and as such we cannot obtain the results for $\hat{\beta}<0$ from the $\hat{\beta}>0$ set-up. We note, however, that, when $\hat{\beta}<0$, as $|\hat{\beta}|$ is increased, the size of the inlet decreases, which has the effect of increased pressure drop in $\hat{\Omega}_{1}$ and thus a reduced pressure drop across the membrane. This consequently decreases the flux through the device. In this paper, we seek a filter configuration that maximizes the processed fluid volume and we will therefore restrict our attention to $\hat{\beta} \geqslant 0$.

\section{A. Governing equations}

The multiphase flow comprises a continuous fluid phase and discrete particles; the phase distribution is described through the corresponding volume fractions $\phi^{f}, \phi^{p}$. We assume the particles to be sufficiently small so as to not disturb the dynamics of the continuous fluid flow. For the purposes of this paper we consider the filtration of neutrally buoyant dust from air, but note that the model and analysis can be readily applied to other applications.

We neglect particle-particle interactions, and the flow dynamics of both phases (fluid and particles) can therefore be described by a single velocity $\hat{\boldsymbol{u}}(\hat{x}, \hat{z}, \hat{t})=(\hat{u}(\hat{x}, \hat{z}, \hat{t}), \hat{w}(\hat{x}, \hat{z}, \hat{t}))$ and pressure $\hat{p}(\hat{x}, \hat{z}, \hat{t})$. We assume there to be a constant volume fraction of particles in $\hat{\Omega}_{1}$ :

$$
\phi^{p}=\phi_{1}^{p} \quad \text { in } \hat{\Omega}_{1} .
$$

The corresponding fluid volume fraction $\phi_{1}^{f}=1-\phi_{1}^{p}$ is therefore also constant. In the subdomain $\hat{\Omega}_{2}$ we assume that no particles are present:

$$
\phi_{2}^{p}=0 \quad \text { in } \hat{\Omega}_{2} .
$$


Hence, the fluid volume fraction in $\hat{\Omega}_{2}$ is $\phi_{2}^{f}=1$. While (2.4) assumes perfect filtration of the particles, we note that it is straightforward to extend this model to account for imperfect filtering with $\phi_{2}^{p} \neq 0$.

The geometry of the system is such that the ratio between the transverse and lateral domain lengths $\epsilon=\hat{X} / \hat{Z}$ is small. Indeed, $\epsilon$ is sufficiently small to result in a small reduced Reynolds number: $\epsilon^{2} \hat{\rho} \hat{W}_{0} \hat{Z} / \hat{\mu}=0.06-0.86$ (see Table I). While we will use the aspect ratio of the entire filter to define $\epsilon$ for mathematical convenience, it is helpful to consider the actual aspect ratio of each fluid domain in the filter in order to interpret the reduced Reynolds number in the system. A better proxy for the height of each fluid domain is $(\hat{X}-\hat{h}) / 2$, which would lead to an effective reduced Reynolds number that is at least four times smaller. As such, we generally expect the effective reduced Reynolds number to be small in each fluid domain, and we proceed by exploiting this smallness. We note that the relative error between the true solution and the approximation will be $O\left(\epsilon^{2} \mathrm{Re}\right)$ and that this lubrication approximation will break down in an $O(\epsilon)$ region from the end of the domain. The flow in $\hat{\Omega}_{1}$ and $\hat{\Omega}_{2}$ is thus modelled through the Stokes equations:

$$
\begin{aligned}
\hat{\mu} \hat{\nabla}^{2} \hat{\boldsymbol{u}}-\hat{\nabla} \hat{p} & =0, \\
\hat{\nabla} \cdot \hat{\boldsymbol{u}} & =0,
\end{aligned}
$$

where $\hat{\mu}$ is the fluid viscosity, which we assume to be constant. We seek solutions $\left(\hat{u}_{1}, \hat{w}_{1}, \hat{p}_{1}\right)$ in $\hat{\Omega}_{1}$ and $\left(\hat{u}_{2}, \hat{w}_{2}, \hat{p}_{2}\right)$ in $\hat{\Omega}_{2}$.

The boundary conditions at the inlet and outlet are

$$
\begin{array}{ll}
\hat{p}_{1}=\hat{p}_{\text {in }} & \text { at } \hat{z}=0, \quad \hat{x} \in[0, \hat{s}(0, \hat{t})], \\
\hat{p}_{2}=\hat{p}_{\text {out }} & \text { at } \hat{z}=\hat{Z}, \quad \hat{x} \in[\hat{m}(\hat{Z})+\hat{h}, \hat{X}] .
\end{array}
$$

At the closed ends in both subdomains, at $\hat{z}=\hat{Z}$ in $\hat{\Omega}_{1}$ and at $\hat{z}=0$ at $\hat{\Omega}_{2}$, we prescribe no-flow boundary conditions:

$$
\begin{array}{lll}
\hat{\boldsymbol{u}}_{1}=\mathbf{0} & \text { at } \hat{z}=\hat{Z}, & \hat{x} \in[0, \hat{s}(\hat{Z}, \hat{t})], \\
\hat{\boldsymbol{u}}_{2}=\mathbf{0} & \text { at } \hat{z}=0, & \hat{x} \in[\hat{m}(0)+\hat{h}, \hat{X}] .
\end{array}
$$

We assume symmetric flow between neighboring modules, which corresponds to the following symmetry conditions:

$$
\begin{aligned}
& \hat{u}_{1}=0, \quad \frac{\partial \hat{w}_{1}}{\partial \hat{x}}=0 \quad \text { at } \hat{x}=0, \\
& \hat{u}_{2}=0, \quad \frac{\partial \hat{w}_{2}}{\partial \hat{x}}=0 \quad \text { at } \hat{x}=\hat{X} .
\end{aligned}
$$

The porous membrane and cake layers provide resistance to the flow. In general, the flow resistance $\hat{R}$ due to a permeable material of thickness $\hat{h}$ and permeability $\hat{k}$ is the reciprocal of the permeance $\hat{\kappa}=\hat{k} / \hat{\mu} \hat{h}: \hat{R}=1 / \hat{\kappa}$. In our problem, the total resistance is the sum of the resistance of the membrane $\hat{R}_{m}$ and that of the cake layer $\hat{R}_{c}$ :

$$
\hat{R}(\hat{z}, \hat{t})=\hat{R}_{m}+\hat{R}_{c}(\hat{z}, \hat{t})=\frac{\hat{\mu} \hat{h}}{\hat{k}_{m}}+\frac{\hat{\mu}[\hat{m}(\hat{z})-\hat{s}(\hat{z}, \hat{t})]}{\hat{k}_{c}},
$$

where $[\hat{m}(\hat{z})-\hat{s}(\hat{z}, \hat{t})]$ is the cake thickness and $\hat{k}_{m}$ and $\hat{k}_{c}$ are the permeabilities of the membrane and cake layer, respectively. Thus we see that the total permeance is

$$
\hat{\kappa}(\hat{z}, \hat{t})=\left\{\frac{\hat{\mu} \hat{h}}{\hat{k}_{m}}+\frac{\hat{\mu}[\hat{m}(\hat{z})-\hat{s}(\hat{z}, \hat{t})]}{\hat{k}_{c}}\right\}^{-1} .
$$

The permeance and pressure difference govern the flow through the porous membrane and cake layers. We impose Darcy flow across the membrane and cake, which provides the outflow boundary 
condition in $\hat{\Omega}_{1}$. This boundary condition is prescribed on $\hat{x}=\hat{s}(\hat{z}, \hat{t})$, which corresponds initially to the membrane surface, $\hat{s}(\hat{z}, 0)=\hat{m}(\hat{z})$, and to the cake front, $\hat{s}(\hat{z}, \hat{t})$, at later times $\hat{t}>0$. The Darcy boundary condition is therefore given by

$$
\hat{\boldsymbol{u}}_{1} \cdot \boldsymbol{n}_{s}=\hat{\kappa}(\hat{z}, \hat{t})\left[\hat{p}_{1}(\hat{s}, \hat{z}, \hat{t})-\hat{p}_{2}(\hat{m}+\hat{h}, \hat{z}, \hat{t})\right] \quad \text { at } \hat{x}=\hat{s}(\hat{z}, \hat{t}),
$$

where $\boldsymbol{n}_{s}$ is the unit normal vector to the cake front.

The corresponding boundary condition for the flow velocity into $\hat{\Omega}_{2}$ from the membrane is given by balancing the flux of fluid through the cake and membrane:

$$
\phi_{1}^{f} \hat{\boldsymbol{u}}_{1}(\hat{s}, \hat{z}, \hat{t})=\hat{\boldsymbol{u}}_{2}(\hat{m}+\hat{h}, \hat{z}, \hat{t}) .
$$

Finally, to close the system of equations, we prescribe no slip on both sides of the membrane:

$$
\begin{aligned}
\hat{\boldsymbol{u}}_{1} \cdot \boldsymbol{t}_{s}=0 & \text { at } \hat{x}=\hat{s}(\hat{z}, \hat{t}), \\
\hat{\boldsymbol{u}}_{2} \cdot \boldsymbol{t}_{m}=0 & \text { at } \hat{x}=\hat{m}(\hat{z})+\hat{h},
\end{aligned}
$$

where $\boldsymbol{t}_{s}$ and $\boldsymbol{t}_{m}$ are, respectively, the unit tangent vectors to the cake front and membrane. While previous work in Ref. [10] describes the appropriate tangential slip-flow boundary condition, subsequent work in Ref. [11] has shown that the inclusion of slip does not significantly affect the flow dynamics and we therefore neglect slip effects here.

\section{B. Caking dynamics}

The model equations presented so far describe the flow dynamics in the subdomains $\hat{\Omega}_{1}$ and $\hat{\Omega}_{2}$. In general there are two key blocking mechanisms in filtration systems: internal blocking and caking. Typically, small particles are filtered out within the membrane depth via internal blocking, while large particles that cannot penetrate into the membrane form a cake layer on the surface. Experimental studies conducted in Ref. [4] indicated that, for the particular application of filtering dust from air using a concertinaed filtration membrane, caking is more prominent than internal blocking, and we therefore focus our study on caking dynamics.

The cake-evolution equation follows from conservation of flux across the moving cake front:

$$
\frac{\partial \hat{s} / \partial \hat{t}}{\sqrt{1+(\partial \hat{s} / \partial \hat{z})^{2}}}=-\phi_{1}^{p} \gamma_{c} \hat{\boldsymbol{u}}_{1}(\hat{s}, \hat{z}, \hat{t}) \cdot \boldsymbol{n}_{s},
$$

where the left-hand side is the normal velocity of the moving cake front and the right-hand side is the influx of particles (see Appendix A for derivation). We have introduced the packing coefficient $\gamma_{c}=$ $\left(\phi_{c}^{p}-\phi_{1}^{p}\right)^{-1}$, where $\phi_{c}^{p}$ is the volume fraction of particles in the cake layer, which is determined by the packing structure within the cake. The packing coefficient $\gamma_{c}$ relates the actual volume of particles in the fluid to the volume of cake they produce. Note that $\gamma_{c}>0$ since $\phi_{c}^{p}>\phi_{1}^{p}$. Initially there will be no cake present:

$$
\hat{s}(\hat{z}, 0)=\hat{m}(\hat{z}) .
$$

\section{Dimensionless model}

We introduce the following dimensionless variables:

$$
\begin{aligned}
& \hat{t}=\tau_{c} t, \quad \hat{z}=\hat{Z}_{z}, \quad \hat{x}=\epsilon \hat{Z} x, \quad \hat{\beta}=\epsilon \beta, \quad \hat{s}=\hat{X} s, \quad \hat{m}=\hat{X} m, \\
& \hat{a}=\hat{X} a, \quad \hat{h}=\hat{X} h, \quad \hat{u}=\epsilon \hat{W}_{0} u, \quad \hat{w}=\hat{W}_{0} w, \quad \hat{p}=\frac{\hat{\mu} \hat{W}_{0}}{\epsilon^{2} \hat{Z}} p+\hat{p}_{\text {out }}, \\
& \hat{\kappa}=\frac{\epsilon^{3} \hat{Z}}{\hat{\mu}} \kappa, \quad \hat{\kappa}_{m}=\frac{\epsilon^{3} \hat{Z}}{\hat{\mu}} \kappa_{m}, \quad \hat{k}_{c}=\epsilon^{3} \hat{Z} \hat{X} k_{c},
\end{aligned}
$$




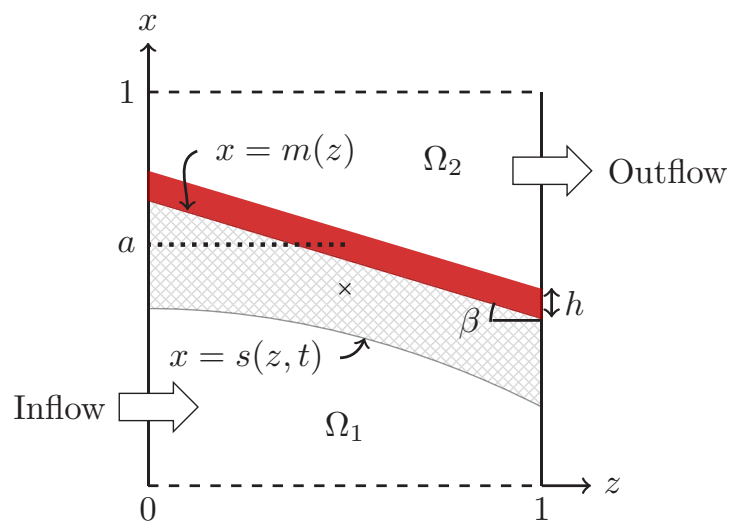

FIG. 2. Dimensionless filtration-module domain $(x, z) \in\{[0,1] \times[0,1]\}$. The membrane and cake layers are schematized in red and gray, respectively, with the evolved cake front denoted by $x=s(z, t>0)$. The membrane center is at $(x, z)=(a, 1 / 2)$ and is specified by the parameter $a$. The membrane angle is given by $\beta$.

where $\tau_{c}=\hat{Z} / \gamma_{c} \hat{W}_{0}$ is the caking timescale, derived from the cake-evolution equation (2.14). Recalling the definition of $\gamma_{c}=\left(\phi_{c}^{p}-\phi_{1}^{p}\right)^{-1}$, we see that changing either the volume fraction of particles in the system, $\phi_{1}^{p}$, or the packing density in the cake (governed by $\phi_{c}^{p}$ ) both affect the timescale $\tau_{c}$ given above. We note that, from (2.16), we can write the velocity scaling in terms of the pressure drop: $\hat{W}_{0}=\epsilon^{2} \hat{Z}\left(\hat{p}_{\text {in }}-\hat{p}_{\text {out }}\right) / \hat{\mu}$.

The membrane angle has been scaled such that $\beta \in[0,1]$. Applying (2.16) to (2.1) yields the dimensionless leading-order membrane position:

$$
m(z)=a-\frac{1}{2} h+\beta\left(\frac{1}{2}-z\right) .
$$

Applying the scalings (2.16) to (2.2) gives the following dimensionless subdomains (shown in Fig. 2):

$$
\begin{aligned}
& \Omega_{1}=\{x \in[0, s(z, t)], z \in[0,1]\}, \\
& \Omega_{2}=\{x \in[m(z)+h, 1], z \in[0,1]\} .
\end{aligned}
$$

Scaling the Stokes equations (2.5) using (2.16) and taking the limit $\epsilon \rightarrow 0$ yields the dimensionless lubrication equations:

$$
\frac{\partial p}{\partial x}=0, \quad \frac{\partial^{2} w}{\partial x^{2}}-\frac{\partial p}{\partial z}=0, \quad \frac{\partial u}{\partial x}+\frac{\partial w}{\partial z}=0 .
$$

The dimensionless inlet and outlet pressures are derived by applying (2.16) to (2.6), which gives

$$
\begin{array}{lll}
p_{1}=1 & \text { at } z=0, & x \in[0, s(0, t)], \\
p_{2}=0 & \text { at } z=1, & x \in[m(1)+h, 1] .
\end{array}
$$

Applying the scalings (2.16) to the no-flow boundary condition (2.7) at the dead-ends results in the loss of the no-slip information, and we are consequently left with the following dimensionless no-penetration boundary condition at the dead-ends:

$$
\begin{array}{lll}
w_{1}=0 & \text { at } z=1, & x \in[0, s(1, t)], \\
w_{2}=0 & \text { at } z=0, & x \in[m(0)+h, 1] .
\end{array}
$$


Enforcing the no-slip condition would require us to rescale into the boundary layer regions near the end caps at $z=0$ and $z=1$, but we expect this to have a small effect on the global flow. The scaled symmetry conditions (2.8) are

$$
\begin{aligned}
& u_{1}=0, \quad \frac{\partial w_{1}}{\partial x}=0 \quad \text { at } x=0, \\
& u_{2}=0, \quad \frac{\partial w_{2}}{\partial x}=0 \quad \text { at } x=1 .
\end{aligned}
$$

The dimensionless Darcy flow [from (2.11)] through the porous membrane and cake is given by

$$
u_{1}=\kappa\left[p_{1}(s, z, t)-p_{2}(m(z)+h, z, t)\right] \quad \text { at } x=s(z, t),
$$

where the dimensionless permeance is

$$
\kappa=\left[\frac{1}{\kappa_{m}}+\frac{m(z)-s(z, t)}{k_{c}}\right]^{-1},
$$

which will evolve over time.

The outflow boundary condition at the membrane (2.12) becomes

$$
\phi_{1}^{f} u_{1}(s, z, t)=u_{2}(m+h, z, t),
$$

and the no-slip conditions (2.13) at the cake front and downstream membrane surface, exploiting the small angle of the membrane, are

$$
\begin{array}{ll}
w_{1}=0 & \text { at } x=s(z, t), \\
w_{2}=0 & \text { at } x=m(z)+h .
\end{array}
$$

Applying (2.16) to the cake-evolution equation (2.14) and taking the limit $\epsilon \rightarrow 0$ yields

$$
\frac{\partial s}{\partial t}=-\phi_{1}^{p} u_{1}(s, z, t)
$$

The corresponding initial condition for the cake position is

$$
s(z, 0)=m(z) .
$$

\section{Model reduction}

The full dimensionless system is given by the lubrication equations (2.19) with boundary conditions (2.19)-(2.25) for each subdomain together with the blocking dynamics described by (2.27)-(2.28). The momentum equation in the $x$ direction (2.19a) gives $p_{1}=p_{1}(z, t)$ and $p_{2}=$ $p_{2}(z, t)$.

In the inflow region $\Omega_{1}$, the lubrication equations (2.19b) and (2.19c) together with the symmetry boundary condition (2.22a) and the condition ensuring no-slip on the cake front (2.26a) can be manipulated to give the velocities in terms of the pressure:

$$
\begin{aligned}
u_{1} & =\frac{1}{2} x\left[\left(p_{1}^{\prime} s^{2}\right)^{\prime}-\frac{1}{3} x^{2} p_{1}^{\prime \prime}\right], \\
w_{1} & =\frac{1}{2} p_{1}^{\prime}\left(x^{2}-s^{2}\right),
\end{aligned}
$$

where ' denotes partial differentiation with respect to $z$. Using (2.29a) in the Darcy flow boundary condition (2.23) we derive the following equation linking the pressures $p_{1}$ and $p_{2}$ :

$$
\frac{1}{3}\left(s^{3} p_{1}^{\prime}\right)^{\prime}=\kappa\left(p_{1}-p_{2}\right) .
$$

The necessary boundary conditions for $p_{1}$ are supplied through the inlet pressure (2.20a) and noflow condition at the closed end (2.21a):

$$
\begin{array}{ll}
p_{1}=1 & \text { at } z=0 \\
p_{1}^{\prime}=0 & \text { at } z=1
\end{array}
$$


We can derive the governing equation of motion for the pressure $p_{2}$ in $\Omega_{2}$ in the same way. We first use the lubrication equations $(2.19 \mathrm{~b})$ and $(2.19 \mathrm{c})$ with the symmetry boundary condition $(2.22 \mathrm{~b})$ and the no-slip condition on the membrane surface (2.26b) to derive

$$
\begin{aligned}
u_{2} & =\frac{1}{2}(x-1)\left(\left\{[m(z)+h-1]^{2}\right\}^{\prime} p_{2}^{\prime}-\frac{1}{3}(x-1)^{2} p_{2}^{\prime \prime}\right), \\
w_{2} & =\frac{1}{2}\left\{(x-1)^{2}-[m(z)+h-1]^{2}\right\} p_{2}^{\prime} .
\end{aligned}
$$

The conservation-of-fluid-flux condition (2.25) with (2.32a) and the equation for $p_{1}$ (2.30) yield a second equation linking the pressures $p_{1}$ and $p_{2}$ :

$$
\frac{1}{3}\left\{[m(z)+h-1]^{3} p_{2}^{\prime}\right\}^{\prime}=\phi_{1}^{f} \kappa\left(p_{1}-p_{2}\right) .
$$

The corresponding boundary conditions are derived from the outlet pressure (2.20b) and no-flow condition at the closed end (2.21b):

$$
\begin{array}{ll}
p_{2}=0 & \text { at } z=1, \\
p_{2}^{\prime}=0 & \text { at } z=0 .
\end{array}
$$

We can use these workings to rewrite the equations for the cake evolution (2.27) and (2.28) in terms of $p_{1}$ and $p_{2}$ :

$$
\frac{\partial s}{\partial t}=-\phi_{1}^{p} \kappa\left(p_{1}-p_{2}\right), \quad s(z, 0)=m(z) .
$$

The problem is therefore reduced to solving the modified Reynolds equations (2.30)-(2.31) for $p_{1}$ in $\Omega_{1}$ and (2.33)-(2.34) for $p_{2}$ in $\Omega_{2}$ coupled with the permeance and cake-evolution equations given by (2.24) and (2.35), respectively. The remaining parameters in the system are the dimensionless midpoint $a$, angle $\beta$, permeance of the membrane $\kappa_{m}$, cake permeability $k_{c}$, and volume fraction of fluid $\phi_{1}^{f}$ in $\Omega_{1}$. In practice the membrane is thin compared with the size of the filter module. We therefore consider the regular limit in which the dimensionless membrane thickness $h \rightarrow 0$ while the membrane permeance $\kappa_{m}$ remains finite. This then removes the geometrical effect of membrane thickness while retaining its resistive properties.

The final reduced system of equations is as follows:

$$
\begin{aligned}
\frac{1}{3}\left(s^{3} p_{1}^{\prime}\right)^{\prime} & =\kappa\left(p_{1}-p_{2}\right),\left.\quad p_{1}\right|_{z=0}=1,\left.\quad p_{1}^{\prime}\right|_{z=1}=0, \\
\frac{1}{3}\left[(m(z)+h-1)^{3} p_{2}^{\prime}\right]^{\prime} & =\phi_{1}^{f} \kappa\left(p_{1}-p_{2}\right),\left.\quad p_{2}\right|_{z=1}=0,\left.\quad p_{2}^{\prime}\right|_{z=0}=0, \\
\kappa & =\left(\frac{1}{\kappa_{m}}+\frac{m-s}{k_{c}}\right)^{-1}, \\
\frac{\partial s}{\partial t} & =-\left(1-\phi_{1}^{f}\right) \kappa\left(p_{1}-p_{2}\right), \quad s(z, 0)=m(z), \\
m & =a+\beta\left(\frac{1}{2}-z\right) .
\end{aligned}
$$

The solution to the model (2.36) provides insight into the flow dynamics and is used to calculate key quantities including the flux of fluid through the filtration-module domain, which is defined by

$$
Q(t)=\left.\int_{0}^{s} \phi_{1}^{f} w_{1}\right|_{z=0} d x=-\left.\frac{1}{3} \phi_{1}^{f}\left(s^{3} p_{1}^{\prime}\right)\right|_{z=0} .
$$

The amount of fluid processed through the device is given by the throughput:

$$
V(t)=\int_{0}^{t} Q(\tau) d \tau
$$


In practice, practitioners will use a filtration membrane at a fixed pressure difference across the module until the flux through the membrane drops such that it is no longer economical to use the filter. We therefore define the end time $T$ to be the time at which the in silico experiment is terminated due to the flux dropping below some critical value $Q_{c}$. A key quantity of interest in this paper will be $V(T)$, the total throughput achieved at the end time.

The key control parameters in the model (2.36) are $\phi_{1}^{f}, a, \beta$, and $\kappa_{m}$. In this paper, we will study the effects of each of these control parameters in optimizing the performance of the filter in turn. In Sec. III we examine how the inclusion of particles influences the flow dynamics in the filter. In Sec. IV we study the effect of the angle and position of the membrane on caking in relation to optimizing performance. In Sec. V we consider how a spatially varying permeance might be utilized to optimize filtration performance.

\section{THE EFFECT OF ADDING PARTICLES TO THE STEADY-STATE SYSTEM}

Previous work in Ref. [5] presents a full analysis of the particle-free steady-state flow through a concertinaed filtration membrane. In this section, we look at the effect of adding particles into the system by considering how the steady-state flow changes with the volume fraction of particles.

To isolate the effect of particle inclusion (i.e., the effect of changing $\phi_{1}^{f}$ ) we focus our attention to a transverse membrane positioned in the center of the domain, by setting $\beta=0$ and $a=0.5$. We further restrict our attention to a steady-state set-up that corresponds to a scenario where the particles are removed on contact with the membrane, so that $s(z, t)=m(z)$ and $\kappa=\kappa_{m}$. Our reduced parameter space then comprises the membrane permeance $\kappa_{m}$ and the volume fraction of fluid in the inflow domain $\phi_{1}^{f}$.

For a membrane of such characteristics operating in this steady state we can derive explicit analytic solutions to (2.36) for the pressure:

$$
\begin{aligned}
p_{10}=\frac{1}{N} & \left\{a^{3}(1-a)^{3}+a^{6} \cosh (M)+(1-a)^{6} \cosh [M(z-1)]\right. \\
& \left.+a^{3}(1-a)^{3} \cosh (M z)-a^{3}(1-a)^{3} M(z-1) \sinh (M)\right\}, \\
p_{20}=\frac{1}{N} & \left\{a^{3}(1-a)^{3}+a^{6} \cosh (M)-a^{3}(1-a)^{3} \cosh [M(z-1)]\right. \\
& \left.-a^{6} \cosh (M z)-a^{3}(1-a)^{3} M(z-1) \sinh (M)\right\},
\end{aligned}
$$

where

$$
M=\sqrt{\frac{3 \kappa_{m}}{a^{3}}+\frac{3 \kappa_{m} \phi_{1}^{f}}{(1-a)^{3}}}, \quad N=\left[\left(a^{6}+(1-a)^{6}\right] \cosh (M)+a^{3}(1-a)^{3}[2+M \sinh (M)] .\right.
$$
by

We consider the total flux (of particles and fluid) in the upstream subdomain $\Omega_{1}$ at $t=0$ defined

$$
Q_{1}=\left.\int_{0}^{a} w_{1}\right|_{z=0} \mathrm{~d} x=-\left.\frac{1}{3}\left(a^{3} p_{10}^{\prime}\right)\right|_{z=0} .
$$

Similarly, we can define the amount of fluid processed through the device by calculating the flux out of the downstream subdomain $\Omega_{2}$ :

$$
Q_{2}=\left.\int_{a}^{1} w_{2}\right|_{z=1} \mathrm{~d} x=\left.\frac{1}{3}\left((a-1)^{3} p_{20}^{\prime}\right)\right|_{z=1} .
$$

From conservation of fluid we have $\phi_{1}^{f} Q_{1}=Q_{2}$.

The analytical result for the steady fluxes as a function of the fluid volume fraction $\phi_{1}^{f}$ is shown in Fig. 3. For a given applied pressure, as the volume fraction of fluid increases, the total flux of particles and fluid in $\Omega_{1}, Q_{1}$, decreases but the total amount of fluid processed through $\Omega_{2}$, 


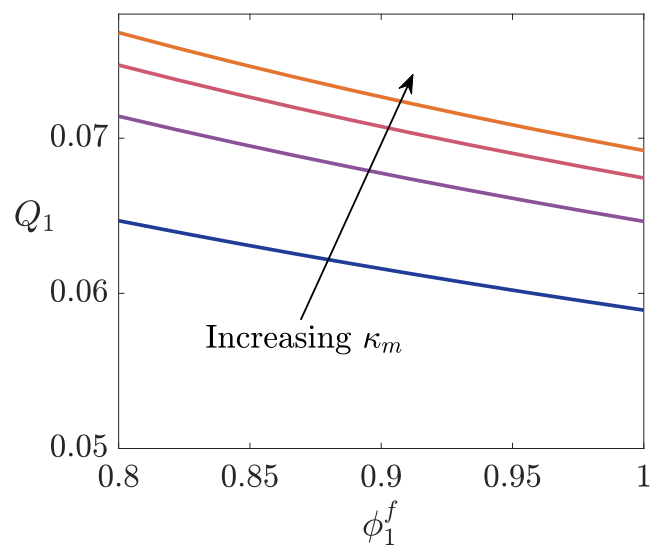

(a)

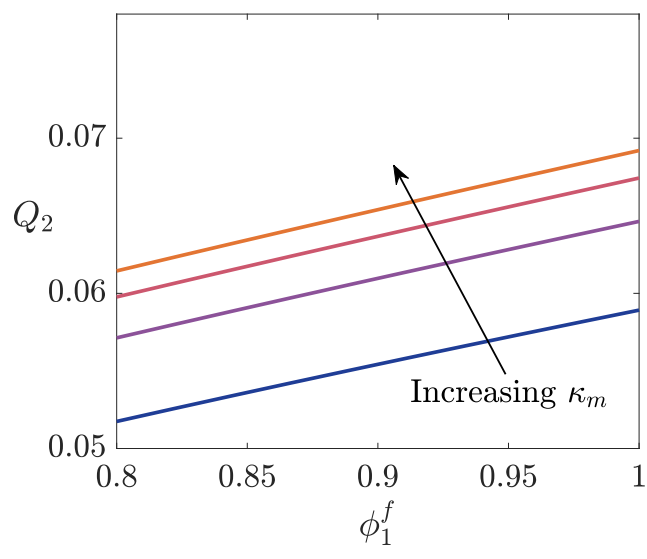

(b)

FIG. 3. Analytical flux in $Q_{1}$ (3.3) in (a) and flux out $Q_{2}$ (3.4) in (b) plotted as functions of the fluid volume fraction for the case when $s(z, t)=m(z)$ and the particles are removed on contact with the membrane. Here $\beta=0$ and $a=0.5$ and $\kappa_{m}=0.5,1,1.5,2$.

$Q_{2}$, increases. Thus, the addition of particles in the system speeds up the flow in the upstream subdomain $\Omega_{1}$, but decreases the total flux of fluid processed out of the device from $\Omega_{2}$. We discuss this effect in further detail for a unidirectional flow in Appendix B in order to uncover the rationale for this observation. Furthermore, we also note that our results show that increasing the membrane permeance $\kappa_{m}$ correspondingly increases the flux (Fig. 3), in agreement with physical intuition. This preliminary study shows that, even before blocking occurs, the initial system of fluid and particles is fundamentally different to the particle-free case.

In this section, we have studied the effect of introducing particles to a concertinaed filter operating in steady state. We now extend our study to explore the effects of control parameters governing the membrane configuration and their effect on the dynamic cake evolution in relation to optimal filtration performance.

\section{THE EFFECT OF MEMBRANE CONFIGURATION}

In this section, we examine the effect of angling and shifting the position of the membrane on the dynamic flux and throughput within the device. As we seek to study the effect of the configuration (namely the parameters $a$ and $\beta$ ), for definiteness we shall assume a constant uniform membrane permeance and cake permeability, $\kappa_{m}=k_{c}=1$, and a constant inflow fluid volume fraction, $\phi_{1}^{f}=0.8$, throughout this section. Choosing a smaller particle volume fraction [together with rescaling time in the cake evolution (2.36d)] would enable the reduction of (2.36) to a quasisteady system of equations, the dynamics of which were studied in Ref. [5]. We therefore choose a large particle volume fraction, which enables us to study the richest behavior capturing both substantial caking and pressure evolution. We expect the model to be valid for our gaseous air-flow system, in a system of liquid the suspension rheology may well need to be accounted for.

\section{A. The effect of membrane angle in relation to the total throughput and end time}

We first explore the effect of angling the membrane. As such, we fix the membrane mid-point to be centered in the domain by setting $a=0.5$, and vary $\beta$. We solve the governing system of equations (2.36) numerically using MATLAB's bvp4c solver, stepping forward in time until the flux 


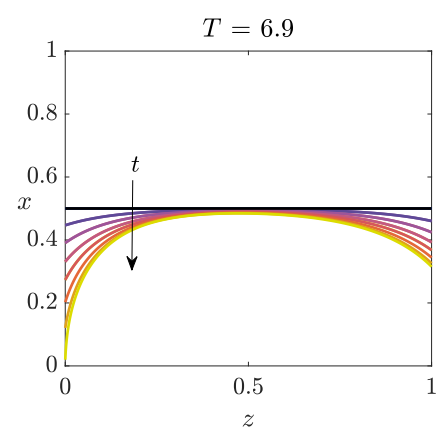

(a)

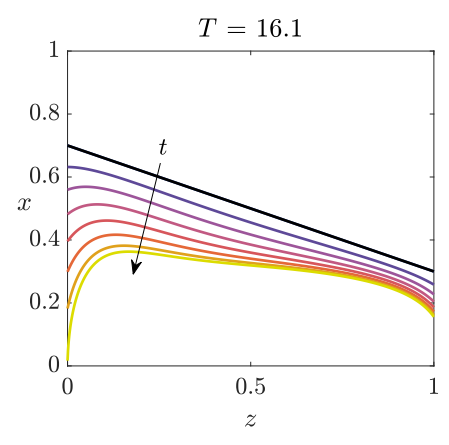

(b)

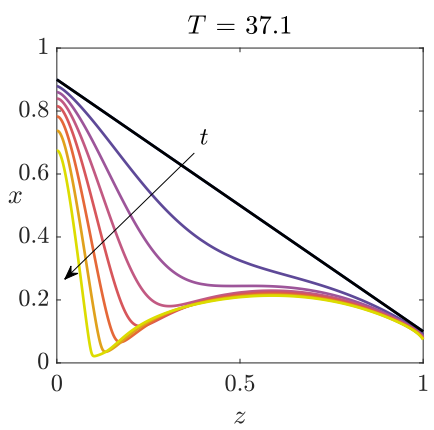

(c)

FIG. 4. Evolution of the cake profile $s(z, t)$ over time found by solving (2.36) for $\beta=0,0.4,0.8$ in panels (a), (b), and (c) respectively, with $a=0.5, \phi_{1}^{f}=0.8, \kappa_{m}=k_{c}=1, Q_{c}=10^{-2}$. The arrows indicated the direction of growth in time, and $T$ is the end time at which the flux $Q$ (2.37) drops below $Q_{c}$. Results are shown for eight time steps equally spaced between $[0, T]$ for each angle tested.

$Q$ (2.37) drops below the critical value $Q_{c}$ which we take to be $Q_{c}=10^{-2}$; this stopping criterion defines the end time $T$ of the simulation.

The resulting cake-evolution profiles for different membrane angles are shown in Fig. 4. Broadly speaking, cake evolution in $\Omega_{1}$ can be considered within three regions: toward the inlet $z=0$, in the center of the membrane, and toward the dead-end $z=1$. We find that angling the membrane has a significant effect on where the cake evolution takes place.

For a horizontal membrane with $\beta=0$, the cake growth predominantly occurs at the domain edges, with the growth at the inlet $z=0$ occurring faster than that at $z=1$ [Fig. 4(a)]. Given that small gaps amplify pressure in lubrication theory, when $a=0.5$, the pressure drop across the membrane is maximal near the inlet when the membrane is horizontal. Since, with $a=0.5$ fixed, the pressure is largest in $\Omega_{1}$ near $z=0$, and the height of $\Omega_{2}$ at $z=0$ is largest for horizontal membranes, the resulting transmembrane pressure drop is maximized near $z=0$. This is what drives the high cake growth near $z=0$. The high cake growth at $z=1$ arises due to the fact that at the dead-end the flow has nowhere to go but through the membrane. The total throughput for the straight membrane is $V(T)=0.29$ and is achieved at $T=6.9$.

For a slightly angled membrane $(\beta=0.4)$, while the cake growth remains focused at the edges, we also observe growth around the center of the membrane [Fig. 4(b)]. Increasing the membrane angle opens the inlet which correspondingly decreases the available space in $\Omega_{2}$ at $z=0$. This increases the pressure in $\Omega_{2}$ and decreases the transmembrane pressure drop at $z=0$. Moreover, angling the membrane increases the available membrane surface area, and decreases the angle that the fluid needs to turn to move normally through the membrane. These combined effects result in the overall cake evolution occurring more uniformly for slightly angled membranes than that for $\beta=0$. The end time for the slightly angled membrane is $T=16.1$, which is more than double that for $\beta=0$, and the corresponding total throughput is also increased to $V(T)=0.80$.

Increasing the membrane angle further $(\beta=0.8)$ intensifies the angled-membrane effects discussed above [Fig. 4(c)]. The increased membrane angle correspondingly increases the inlet size into $\Omega_{1}$ and decreases the available space in $\Omega_{2}$, thus the transmembrane pressure difference at $z=0$ is reduced and we see very little blocking near the inlet. The increased membrane surface area and orientation of the membrane result in an increased flow across the center of the membrane where we see prominent blocking. Consequently most of the fluid and particles are processed along the length of the membrane, and thus there is little filtration at the dead-end, $z=1$, and so we see reduced blocking. Thus for significantly angled membranes, we observe a distinct cake-evolution profile with the growth focused at the center of the membrane. The corresponding end time $T=37.1$ 


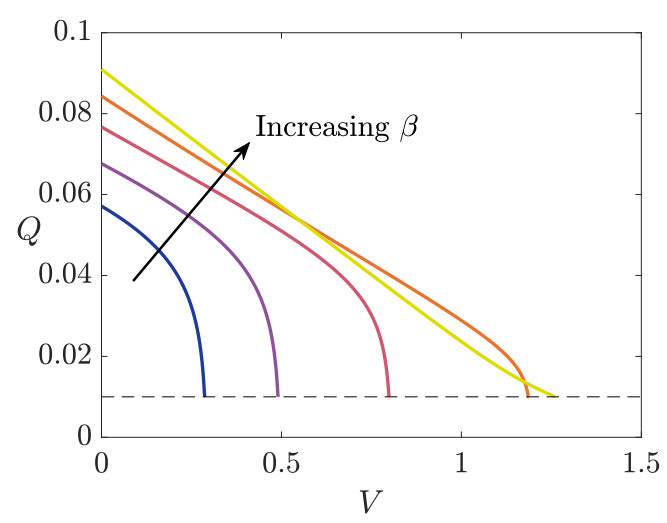

(a)

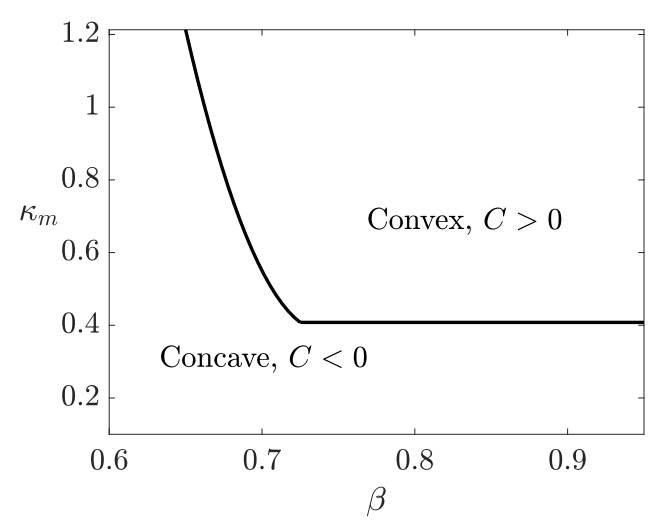

(b)

FIG. 5. Flux-throughput relationship resulting from solving the system of Eqs. (2.36) and calculating the flux $Q$ [Eq. (2.37)] and throughput $V$ [Eq. (2.38)] for $a=0.5, \phi_{1}^{f}=0.8, \kappa_{m}=1, Q_{c}=10^{-2}$, and $\beta=0,0.2,0.4,0.6,0.8$ in (a). Convexity $C(4.1)$ of the flux-throughput curves indicated through $\operatorname{sgn}(C)$ in the $\left(\kappa_{m}, \beta\right)$ domain in $(b)$.

and total throughput $V(T)=1.26$ are significantly larger than found to be for $\beta=0$ and $\beta=0.4$ (Fig. 4). These results show the effect of angling the membrane away from horizontal and indicate that angling the membrane results in the flux remaining above $Q_{c}$ for longer, thus prolonging the lifetime of the filter and increasing the amount of fluid processed by the filter.

\section{B. The effect of membrane angle on the flux-throughput curve}

In practice, a standard tool to analyze the performance of a filter is the visualization of the flux $(Q)$ and throughput $(V)$ relationship over time. The classic interpretation of these visualizations is that concave curves, i.e., those for which $\mathrm{d}^{2} Q / \mathrm{d} V^{2}<0$, correspond to blocking due to caking, and convex curves, i.e., those for which $\mathrm{d}^{2} Q / \mathrm{d} V^{2}>0$, represent internal blocking [12]. We present the flux-throughput curves for varying membrane angles in Fig. 5(a). For straight to slightly angled membranes the flux-throughput curve is clearly concave. For larger values of $\beta$, however, the curve becomes convex. We examine this effect more closely by testing the convexity as a function of the membrane permeance $\kappa_{m}$ and angle $\beta$. We do this by fitting a second-order polynomial to the function $Q(V)$ to produce the interpolated flux function $\tilde{Q}(V)$. We then define the convexity measure by

$$
C=\frac{\mathrm{d}^{2} \tilde{Q}}{\mathrm{~d} V^{2}}
$$

where $C<0$ yields a concave function and $C>0$ a convex function.

For small values of the membrane permeance $\left(\kappa_{m}<0.4\right)$ we find that the convexity measure remains negative for all membrane angles $\beta$ indicating concave flux-throughput curves [Fig. 5(b)]. For increased values of $\kappa_{m}$, however, the flux-throughput curves are convex for large values of $\beta$. This analysis yields the values $\kappa_{m}(\beta)$ at which the convexity switches. We find that, for certain sets of system parameters, the flux-throughput curves will be convex for blocking by caking only. These results show that interpreting the dominant blocking mechanism by the convexity of the flux-throughput curves can be inaccurate when the membrane is angled.

To understand what is driving this convexity switch it is helpful to consider what convexity means for filtration curves, and specifically what this means for the dominant blocking location. First, note that a straight flux-throughput profile would arise when blocking occurs uniformly in time. Concave 
flux-throughput curves arise when the membrane blocks faster at later times, which will occur when blocking occurs predominantly at the entrance of the filter. We observed this happening for less angled membranes with a lower $\beta$ [Figs. 4(a) and 4(b)]. Convex flux-throughput curves, however, arise when blocking occurs faster initially then slows. This corresponds to blocking occurring across a large region of the membrane at later times. In this scenario, the flow is eventually reduced by the geometry of the large cake layer in the upstream subdomain. We observed this behavior for large $\beta$ values [Fig. 4(c)].

\section{The optimal membrane configuration}

We now investigate the effect of additionally shifting the membrane (by varying $a$ ), with the objective of obtaining the optimal membrane configuration according to a given requirement. We explore the full angle domain $\beta \in[0,1]$ and position domain $a \in[\beta / 2,1-\beta / 2]$. We seek numerical solutions but note that when either end of the membrane is close to a corner of the domain, the behavior is difficult to resolve numerically. Thus, we slightly reduce our parameter domain to $\beta \in\left[0,1-\delta_{\beta}\right]$ and $a \in\left[\beta / 2+\delta_{a}, 1-\left(\beta / 2+\delta_{a}\right)\right]$ for some small $\delta_{\beta}, \delta_{a}>0$, and extrapolate our results to the full domains. For the results presented in this section we take $\delta_{\beta}=0.05$ and $\delta_{a}=0.01$.

There are various performance measures associated to filtration optimisation with the choice of measure depending on the industrial application. We choose to focus our attention to maximizing the total throughput $V(T)$ and the average flux $\bar{Q}(T)=V(T) / T$. Note that since the instantaneous flux decreases in time, maximizing $\bar{Q}$ corresponds to maximizing the amount of fluid processed over the shortest time period. To examine how the optimal configuration depends on the choice of metric, we also consider a one-parameter family of measures in the form of a convex combination of the two metrics:

$$
E(\alpha)=\alpha V(T)+(1-\alpha) \bar{Q}(T),
$$

for some constant $\alpha \in[0,1]$. While we present results for the chosen metrics only, the model presented in this paper can be used for any choice of optimization metric.

We show results for our performance metrics in Fig. 6. We see that the total throughput is minimized for a horizontal membrane with $\beta=0$ positioned toward the top and bottom of the domain, where $a=1$ and $a=0$, respectively [Fig. 6(a)]. This corresponds to a filtration device in which the concertinaed membranes have minimal surface area and are close to touching. The maximum total throughput is achieved for $\beta \approx 0.2$ and $a \approx 0.7$ where the membrane is slightly angled and positioned off-center upwards toward $x=1$ [Fig. 6(a)]. This configuration highlights the trade-off between maximizing the membrane area (by angling the membrane) and the transmembrane pressure drop (by shifting the membrane off-center) that was identified in previous work analyzing the analogous steady-state system [5]. We find that to maximize the total throughput, it is more important to maximize the transmembrane pressure drop than the available membrane area.

The average flux is similarly minimized for straight membranes where the concertina pleats close up with $\beta=0$ and $a=0$ or $a=1$ [Fig. 6(b)]. The average flux is maximized for $\beta \approx 0.7$ and $a \approx 0.4$. This is associated to membranes significantly angled and positioned off-center toward the lower boundary at $x=0$. These results are also due to the trade-off between maximizing the membrane area and the transmembrane pressure drop; for maximizing average flux, we find it is more important to maximize the membrane area.

The distinction between the configurations that maximize $V(T)$ and $\bar{Q}$ is made clear when we consider the configurations that maximize $E(\alpha)$ [Fig. 6(c)]. Our results show that the two optimal configurations discussed above are the only optimal configurations for $\alpha \in[0,1]$. For small values of $\alpha$ the optimal configuration agrees with that which maximizes the average flux. As the value of $\alpha$ is increased, the optimal configuration switches at (and remains fixed for all values of $\alpha$ greater than) the critical value of $\alpha \approx 0.02$ to coincide with the configuration that maximizes the total throughput. The critical value of $\alpha \approx 0.02$ is notably small since there is a large difference between the magnitudes of the metrics $V(T)$ and $\bar{Q}$. Thus we conclude that for particular applications for 


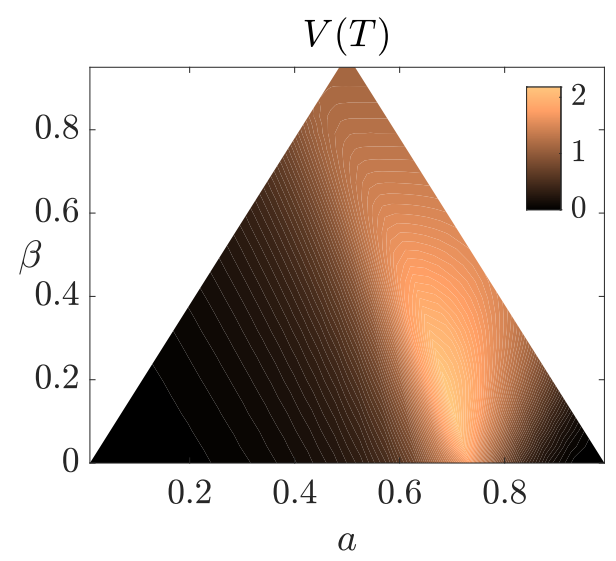

(a)

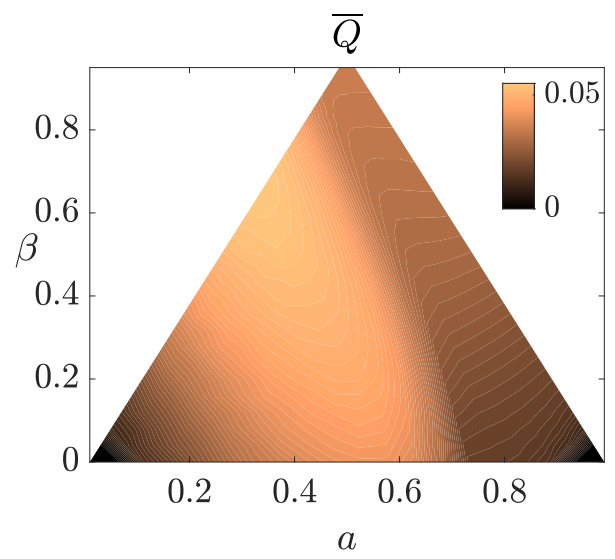

(b)

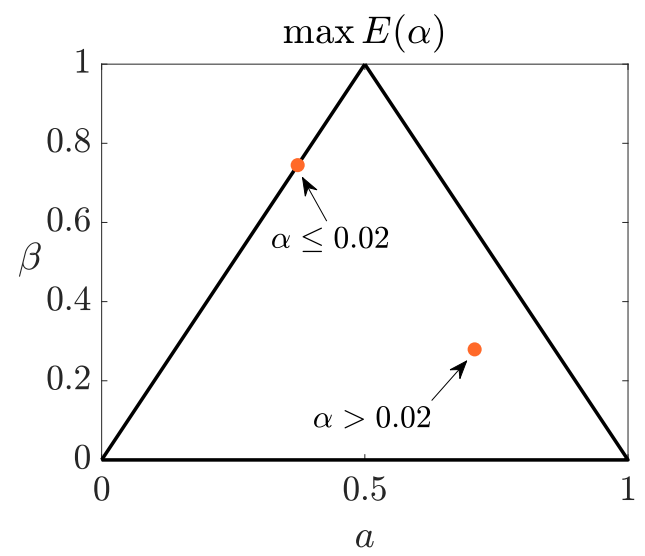

(c)

FIG. 6. The total throughput $V(T)$ (2.38) in (a), $\bar{Q}=V(T) / T$ in (b), and the configuration that maximizes $E(\alpha)$ (4.2) in (c) calculated from numerical solutions to (2.36) with $\beta \in[0,1], a \in[\beta / 2,1-\beta / 2], \kappa_{m}=1$, $\phi_{1}^{f}=0.8$, and $Q_{c}=10^{-2}$.

which it is preferable to maximize the total amount of fluid processed, irrespective of the time it takes, the optimal configuration is a slightly angled membrane positioned off-center upwards toward $x=1$. For applications where it is important to maximize the amount of fluid processed whilst minimizing the time of operation, the optimal configuration is a membrane that is significantly angled and positioned off-center toward the lower boundary at $x=0$. The optimal angle and position in both cases are provided by our model.

\section{THE EFFECT OF A SPATIALLY VARYING PERMEANCE}

We have so far considered a constant membrane permeance $\kappa_{m}$. In this section we relax this assumption to investigate the effect of a spatially varying permeance. This is motivated by the observation that a spatially varying permeance may be chosen to ensure uniform cake growth, which optimizes the use of membrane area and minimizes the effect of the changing geometry on introducing resistance to the flow. A nonuniform permeance could be achieved by varying 
either the membrane thickness in space or the pore distribution. The latter of these is becoming increasingly achievable through new technologies that enable practitioners to engineer a nonuniform pore distribution.

Although, in theory, we can derive the membrane permeance $\kappa_{m}(z, t)$ that yields uniform cake growth for all time, in practice it is difficult to vary the permeance in time. Therefore we choose to find the time-steady permeance $\kappa_{m}(z)$ that yields uniform cake growth initially. Steady uniform cake growth is achieved for a permeance profile that generates a constant flow at the membrane front at $t=0$ :

$$
u_{1}(x, z, 0)=\text { const }=U_{m} \quad \text { at } x=m(z) .
$$

Initially, $s(z, 0)=m(z)$ and so $\kappa(z, 0)=\kappa_{m}(z)$ using (2.36c). Using (5.1) in the Darcy-flow boundary condition (2.23) at the membrane, and noting that we have $p_{i}=p_{i}(z, t)$ for $i=1,2$ from the lubrication equation $(2.19 \mathrm{a})$, we then find

$$
\kappa_{m}(z)=\frac{U_{m}}{p_{1}(z, 0)-p_{2}(z, 0)} .
$$

We use (5.2) to simplify the governing equations for the pressure (2.36a)-(2.36b), which decouple to give

$$
\begin{aligned}
& \frac{1}{3}\left[m(z)^{3} p_{1}^{\prime}(z, 0)\right]^{\prime}=U_{m},\left.\quad p_{1}\right|_{z=0}=1,\left.\quad p_{1}^{\prime}\right|_{z=1}=0, \\
& \frac{1}{3}\left[(m(z)+h-1)^{3} p_{2}^{\prime}(z, 0)\right]^{\prime}=\phi_{1}^{f} U_{m},\left.\quad p_{2}\right|_{z=1}=0,\left.\quad p_{2}^{\prime}\right|_{z=0}=0 .
\end{aligned}
$$

Equations (5.3) admit exact analytical solutions for $p_{1}$ and $p_{2}$ :

$$
\begin{aligned}
& p_{1}(z, 0)=1+\frac{12 U_{m} z[2 a(z-2)+\beta(3 z-2)]}{(2 a+\beta)^{2}(2 a+\beta-2 \beta z)^{2}} \\
& p_{2}(z, 0)=\frac{12 U_{m}(z-1) \phi_{1}^{f}[2 a(z+1)+\beta-(3 \beta+2) z-2]}{(-2 a+\beta+2)^{2}(2 a+\beta-2 \beta z-2)^{2}} .
\end{aligned}
$$

From (5.2) we see that if there exists a zero pressure difference $\Delta p=p_{1}(z, 0)-p_{2}(z, 0)=0$ anywhere along the membrane there will be an infinite permeance at that point, which corresponds to physical holes in the membrane. Note that we have introduced the, so far, unspecified parameter $U_{m}$. For each position and angle $(a, \beta)$ there exists a bounded range of values of $U_{m}$ for which $\Delta p$, calculated from (5.4), is such that $\Delta p>0$ for all $z$. We first see that $U_{m}$ is bounded from below by $U_{m}=0$ for which $p_{1}=1$ and $p_{2}=0$ from (5.4). To find an upper bound, we find the maximum value of $U_{m}$ for which min $\Delta p>0$ holds at $t=0$ :

$$
U_{m}^{*}(a, \beta)=\max U_{m} \quad \text { for which } \quad \Delta p(z, 0 ; a, \beta)>0 \text { for all } z .
$$

This gives the bounded range $U_{m} \in\left[0, U_{m}^{*}\right]$ for each $(a, \beta)$ pairing. The contour plot for $U_{m}^{*}$ in $\beta \in\left[0,1-\delta_{\beta}\right]$ and $a \in\left[\beta / 2+\delta_{a}, 1-\left(\beta / 2+\delta_{a}\right)\right]$ for $\delta_{\beta}=0.1$ and $\delta_{a}=0.04$ is given in Fig. 7 (a). We find that for a membrane positioned toward the corners of the domain, at $a=0$ or $a=1$, a very slow flow $U_{m}^{*} \ll 1$ is required for $\Delta p>0$. For more centered membranes $(a \approx 0.5), \Delta p>0$ can be achieved for faster flows. However, crucially, for the full $(a, \beta)$ parameter space tested, $U_{m}^{*}$ is a small parameter, and thus uniform cake growth is only achieved for small fluxes through the domain.

We present the resulting permeance profiles $\kappa_{m}(z)$ in Fig. 7(b) for a range of membrane angles. Note that the observed dimensionless permeance is $O\left(10^{-2}\right)$, which, when rescaled using the parameter values in Table I and the scalings (2.16), gives a membrane permeability of $O\left(10^{-11} \mathrm{~m}^{2}\right)$. The derived dimensional membrane permeability is comparable to that of an industrial filter with a pleated configuration (with no dead-ends) produced by Pall Corporation [8].

For horizontal and slightly angled membranes the permeance profile that ensures constant velocity at the membrane is concave [Fig. 7(b)]. What this means in practice is that for horizontal (or close to horizontal) membranes we should choose a membrane with a higher permeance toward the center of the membrane to obtain a constant velocity across the entire membrane. This is because, 


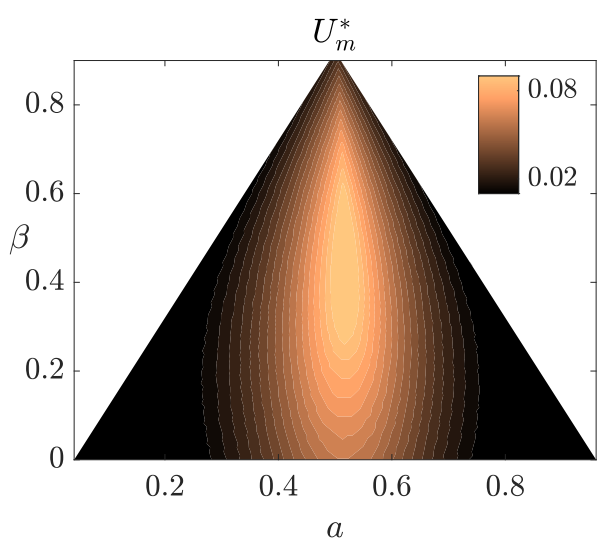

(a)

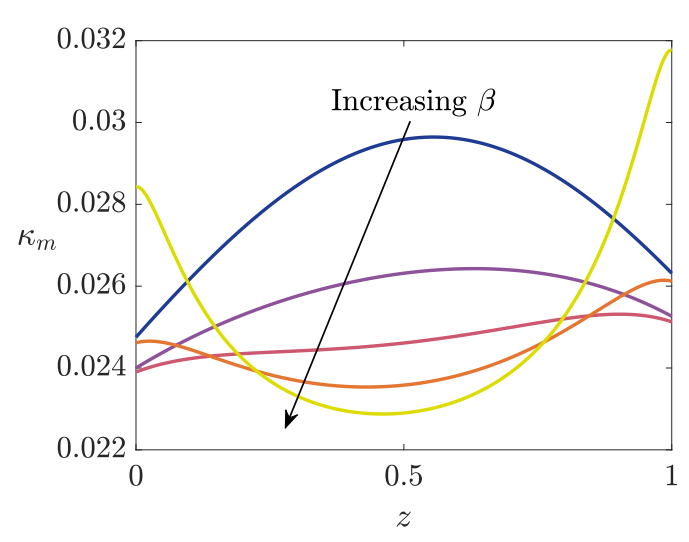

(b)

FIG. 7. The upper bound of the constant velocity $U_{m}^{*}$ calculated in (5.5) using analytical pressure profiles (5.4) and $\beta \in[0,1]$ and $a \in[\beta / 2,1-\beta / 2]$ given in (a). The nonuniform membrane permeance profiles $\kappa_{m}(z)$ (5.2) calculated using (5.4) with $\beta=0,0.2,0.4,0.6,0.8$ at $t=0$ in (b) for $a=0.5$ and $U_{m}=0.02$. Both figures use $\phi_{1}^{f}=0.8$.

as discussed in Sec. IV A, for membranes that are close to horizontal the transmembrane pressure drop is highest at the entrance $z=0$. The transmembrane pressure drop is also increased near the dead-end, $z=1$, because the flow has nowhere to go but through the membrane. Thus, to avoid the cake building up predominantly at the device entrance and at the dead-end one should reduce the permeance at these two locations [Figs. 4(a) and 4(b)]. This will result in a more uniform growth across the entire filter.

As we increase the angle of the membrane the concavity of the permeance profile decreases, and for significantly angled membranes the membrane profile is strongly convex [Fig. 7(b)]. In this case, we should therefore increase the permeance at the edges of the membrane and decrease the permeance toward to the center. This is because, for significantly angled membranes, we have opened up the inlet and increased the available surface area of the membrane. As discussed in Sec. IV A, the transmembrane pressure drop is correspondingly maximized toward the center of the membrane. Thus the cake build-up mainly occurs in the middle of the membrane [Fig. 4(c)], and so increasing the permeance at this point will ensure constant cake growth.

\section{CONCLUSIONS}

We have examined caking dynamics in a concertinaed ceramic filtration membrane. Our work builds on the previous work of Ref. [5], which consisted of a study of the steady particle-free flow through a concertinaed filtration membrane. In this paper, we further developed the model to study the flow of a particle-laden fluid mixture through the same filtration device and derive the appropriate model for external cake build-up on the membrane surface. We modelled the flow of the fluid mixture on either side of the membrane using the Stokes equations and the flow through the porous membrane and cake layers using Darcy's law. By systematically reducing the model equations, we simplified the flow problem to solving coupled lubrication equations, which account for the moving boundary associated with blocking, in the upstream and downstream subdomains on either side of the membrane.

Practitioners use flux-throughput curves to determine the nature of blocking within a filter, with concave and convex curves identified, respectively, with caking and internal blocking. We found, however, that when a centered membrane is angled caking dynamics can result in a convex 
flux-throughput curve. This result highlights potential misinterpretations that may result from using these curves to identify the governing blocking mechanism.

The overarching aim of this paper is to understand the effect of the membrane configuration on the filtration performance, which is determined by the particular measure of choice. As this metric will vary for different industrial applications, we considered a one-parameter family of metrics in this paper. The two extreme points of this family correspond to maximization of the total throughput and average flux, respectively, while points in between correspond to linear combinations of the two properties. For the particular set-up of an infinitely thin membrane with unit permeance, the configuration that maximizes the total throughput is a slightly angled membrane positioned off-center upwards in the filtration-module domain. This configuration is distinct to that which optimizes the average flux, which is a significantly angled membrane positioned off-center toward the lower boundary of the domain.

We also explored the derivation of a spatially varying permeance corresponding to an initially uniform cake growth. We found that, to ensure a uniform cake growth, one requires a very small flux through the device initially. Thus, in practice, a nonuniform permeance will not be a useful tool when maximizing the average flux through the domain (and consequently nor will it be useful to maximize the total throughput), but it may be relevant for different optimization metrics. While our analysis was motivated by optimizing the use of membrane area in a concertinaed filtration device, analogous methodology may be applied to the development process of composite filtration membranes; in which a thin porous membrane of uniform thickness is applied to a porous support material [13].

In this paper, we introduced a methodology to derive the membrane permeance that ensures uniform initial deposition. We could instead have explored the more involved problem seeking a membrane permeance that leads to a uniform deposition of cake after a finite amount of time. In this context, we would need to solve an inverse problem for the initial membrane permeance that provides uniform cake profile at a later time. While the actual formulation of such an inverse problem would be challenging in its own right, we expect the limitations on the physical parameter regimes to be similar, if not more restricted, to those found for initial uniform cake growth discussed above.

In this paper we have introduced a mathematical framework for modeling caking in a directflow filtration device. Smart Separations Ltd are a start-up company who have developed a directflow filtration device similar to that discussed in this paper. Motivated by insight provided by this company, we focused on the effect of caking dynamics on filtration performance. An interesting extension of the work presented here would be to explore the additional blocking mechanism of internal blocking.

The model formulation and results presented in this paper may be readily used by practitioners: For a specified application, this work provides understanding of the device configuration that optimizes filtration performance.

\section{ACKNOWLEDGMENTS}

We are grateful to Meredith Ellis for her work in the project conception. We are grateful to Enrique Ruiz-Trejo and Sotiria Tsochataridou from Smart Separations Ltd for their helpful insight into the filtration device. V.E.P. is grateful to The Royal Society, Grant No. URF/R/191008 for funding. I.M.G. is grateful to the Royal Society for funding through a University Research Fellowship.

\section{APPENDIX A}

The cake front is an interface separating the upstream domain $\hat{\Omega}_{1}$ and the cake layer $\hat{\Omega}_{c}=\{\hat{x} \in$ $[\hat{s}(\hat{z}, \hat{t}), \hat{s}(\hat{z}, \hat{t})+\hat{h}], \hat{z} \in[0,1]\}$. The position of this interface can be given implicitly by $\hat{F}(\hat{x}, \hat{z}, \hat{t})=$ $\hat{x}-\hat{s}(\hat{z}, \hat{t})=0$. The normal velocity of this moving boundary $\hat{v}_{n}:=\hat{\boldsymbol{v}} \cdot \boldsymbol{n}_{s}$ can be written as

$$
\hat{v}_{n}=-\frac{\partial \hat{F} / \partial \hat{t}}{|\nabla \hat{F}|} .
$$




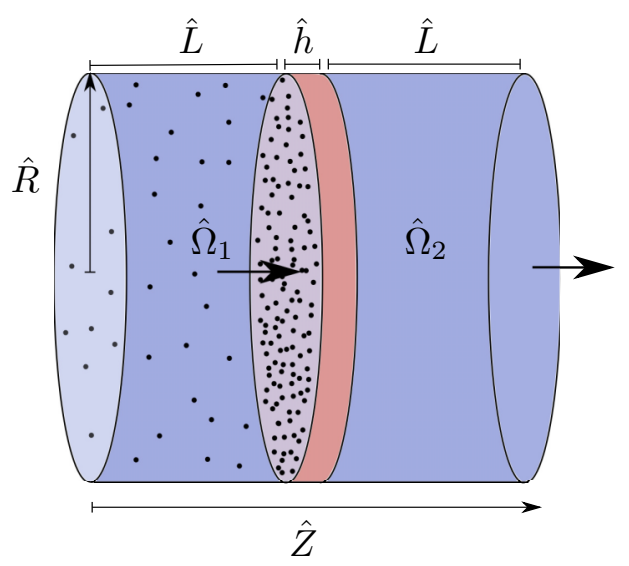

FIG. 8. Schematic of the dimensional unidirectional pipe flow through a membrane. Particles are filtered out by the membrane drawn in pink in the center of the pipe.

Imposing conservation of flux across this moving boundary yields

$$
\hat{\boldsymbol{Q}}_{1} \cdot \boldsymbol{n}_{s}-\hat{v}_{n} \hat{c}_{1}=\hat{\boldsymbol{Q}}_{c} \cdot \boldsymbol{n}_{s}-\hat{v}_{n} \hat{c}_{c},
$$

where $\hat{\boldsymbol{Q}}_{1}$ and $\hat{\boldsymbol{Q}}_{c}$ are the particle fluxes within the upstream and cake domains, respectively, and $\hat{c}_{1}$ and $\hat{c}_{c}$ are the concentrations of particles within the upstream and cake domains, respectively.

The concentration of particles in $\hat{\Omega}_{1}$ and $\hat{\Omega}_{c}$ are related through

$$
\frac{\hat{c}_{c}}{\phi_{c}^{p}}=\frac{\hat{c}_{1}}{\phi_{1}^{p}}
$$

where $\phi_{c}^{p}$ and $\phi_{1}^{p}$ are, respectively, the volume fractions of particles in $\hat{\Omega}_{c}$ and in $\hat{\Omega}_{1}$. We have taken the intrinsic particle volume to be the same value in both phases. Neglecting diffusion in $\hat{\Omega}_{1}$, we can write the particle flux as $\hat{\boldsymbol{Q}}_{1}=\hat{c}_{1} \hat{\boldsymbol{u}}_{1}$. Moreover, we assume particles are not advected within the cake layer, and hence $\hat{\boldsymbol{Q}}_{c}=\mathbf{0}$. Using these values for the particle fluxes in (A2), together with (A3), we obtain the following expression for the normal velocity of the cake layer in terms of the system variables:

$$
\hat{v}_{n}=-\frac{\phi_{1}^{p}}{\phi_{c}^{p}-\phi_{1}^{p}} \hat{\boldsymbol{u}}_{1} \cdot \boldsymbol{n}_{s}
$$

Substituting $\hat{v}_{n}$ (A4) into (A1) and rewriting in terms of the cake front position $\hat{s}(\hat{z}, \hat{t})$ gives (2.14) which, together with the initial condition (2.15), yields the cake evolution equation.

\section{APPENDIX B}

In this Appendix we consider a unidirectional pipe flow and include a porous membrane in the middle of the domain. The aim of this study is to understand the effect to the flow when particles are added to a system. We consider a unidirectional pipe flow to enable analytical insight.

We consider a cylindrical pipe of radius $\hat{R}$ and length $\hat{Z}$ as shown in Fig. 8. We assume axisymmetry and use cylindrical coordinates $(\hat{r}, \hat{z})$ to specify the geometry: We position a porous membrane of thickness $\hat{h}$ centered along the pipe at $\hat{z}=\hat{L}$, where $\hat{Z}=2 \hat{L}+\hat{h}$; the domain is then divided into the upstream and downstream subdomains,

$$
\begin{aligned}
& \hat{\Omega}_{1}=\{\hat{r} \in[0, \hat{R}], \hat{z} \in[0, \hat{L}]\}, \\
& \hat{\Omega}_{2}=\{\hat{r} \in[0, \hat{R}], \hat{z} \in[\hat{L}+\hat{h}, \hat{Z}]\} .
\end{aligned}
$$


The fluid mixture is made up of a continuous fluid phase and discrete particles to be filtered. We assume a constant volume fraction of fluid $\phi_{1}^{f}$ and volume fraction of particles $\phi_{1}^{p}=1-\phi_{1}^{f}$ in $\hat{\Omega}_{1}$. We further assume that there is perfect filtration by the membrane such that the downstream subdomain $\hat{\Omega}_{2}$ comprises only the fluid phase and as such the volume fraction of fluid is $\phi_{2}^{f}=1$.

The flow is driven by a fixed pressure drop across the pipe $\Delta \hat{p}=\hat{p}_{\text {in }}-\hat{p}_{\text {out }}>0$, with $\hat{p}=\hat{p}_{\text {in }}$ at $\hat{z}=0$ and $\hat{p}=\hat{p}_{\text {out }}$ at $\hat{z}=\hat{Z}$. We denote the distinct pressure drops across $\hat{\Omega}_{1}$, the membrane, and $\hat{\Omega}_{2}$ by $\Delta \hat{p}_{1}, \Delta \hat{p}_{m}$, and $\Delta \hat{p}_{2}$, respectively. The total pressure drop across the pipe is therefore

$$
\Delta \hat{p}=\Delta \hat{p}_{1}+\Delta \hat{p}_{m}+\Delta \hat{p}_{2}
$$

We assume a steady, laminar, incompressible Poiseuille flow in $\hat{\Omega}_{1}$ and $\hat{\Omega}_{2}$, and can therefore express the total inflow flux of fluid and particles $\hat{Q}_{1}$ in $\hat{\Omega}_{1}$ and total outflow flux of fluid $\hat{Q}_{2}$ in $\hat{\Omega}_{2}$ in the following way:

$$
\begin{aligned}
& \hat{Q}_{1}=\frac{\pi \hat{R}^{4} \Delta \hat{p}_{1}}{8 \hat{\mu} \hat{L}}, \\
& \hat{Q}_{2}=\frac{\pi \hat{R}^{4} \Delta \hat{p}_{2}}{8 \hat{\mu} \hat{L}} .
\end{aligned}
$$

Furthermore, we prescribe Darcy flow across the membrane to write the flux through the membrane as:

$$
\hat{Q}_{m}=\pi \hat{R}^{2} \hat{\kappa}_{m} \Delta \hat{p}_{m}
$$

where $\hat{\kappa}_{m}$ is the permeance of the membrane.

We nondimensionalize the system by introducing the following scalings:

$$
\begin{array}{cll}
\hat{r}=\hat{R} r, \quad \hat{z}=\delta \hat{R} z, & \hat{p}=\frac{\hat{\mu} \hat{W}}{\delta^{2} \hat{L}} p+\hat{p}_{\text {out }}, & \hat{\kappa}_{m}=\frac{\hat{R}^{2}}{8 \hat{L} \hat{\mu}} \kappa_{m}, \\
\hat{Q}_{1}=\frac{\pi \hat{W} \hat{R}^{2}}{\delta^{4}} Q_{1}, & \hat{Q}_{2}=\frac{\pi \hat{W} \hat{R}^{2}}{\delta^{4}} Q_{2}, & \hat{Q}_{m}=\frac{\pi \hat{W} \hat{R}^{2}}{\delta^{4}} Q_{m},
\end{array}
$$

where $\delta=\hat{L} / \hat{R}$. Moreover, the velocity scaling $\hat{W}$ is given in terms of the pressure drop:

$$
\hat{W}=\frac{\delta^{2} \hat{L}}{\hat{\mu}}\left(\hat{p}_{\text {in }}-\hat{p}_{\text {out }}\right) .
$$

We choose a short, wide domain with $\delta \ll 1$. This enables a closer comparison between the unidirectional flow system in this Appendix and the nonunidirectional lubrication flow system presented in the body of the paper and discussed in Sec. III.

Applying the scalings (B5) to (B3) and (B4) yields the dimensionless fluxes in terms of the dimensionless pressure drops:

$$
Q_{1}=\Delta p_{1}, \quad Q_{m}=\kappa_{m} \Delta p_{m}, \quad Q_{2}=\Delta p_{2} .
$$

Using (B7) in (B3) together with conservation of fluid: $\phi_{1}^{f} Q_{1}=Q_{m}=Q_{2}$ we can find the inflow and outflow fluxes in terms of the total pressure drop $\Delta p$ :

$$
\begin{aligned}
Q_{1} & =\frac{\Delta p}{1+\phi_{1}^{f}+\frac{\phi_{1}^{f}}{\kappa_{m}}}, \\
Q_{2} & =\frac{\Delta p}{1+\frac{1}{\phi_{1}^{f}}+\frac{1}{\kappa_{m}}} .
\end{aligned}
$$




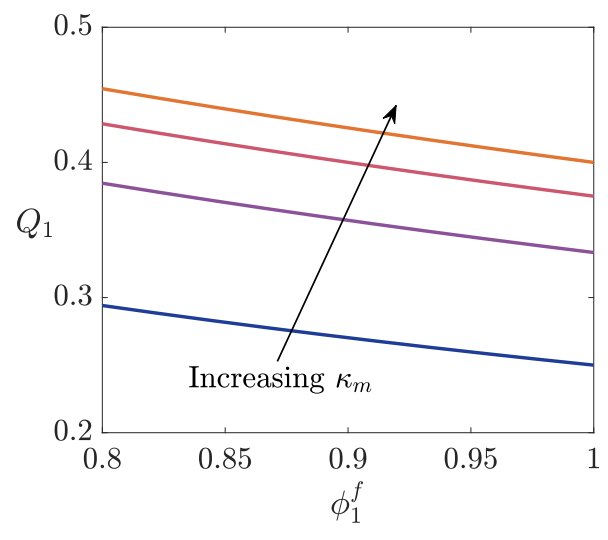

(a)

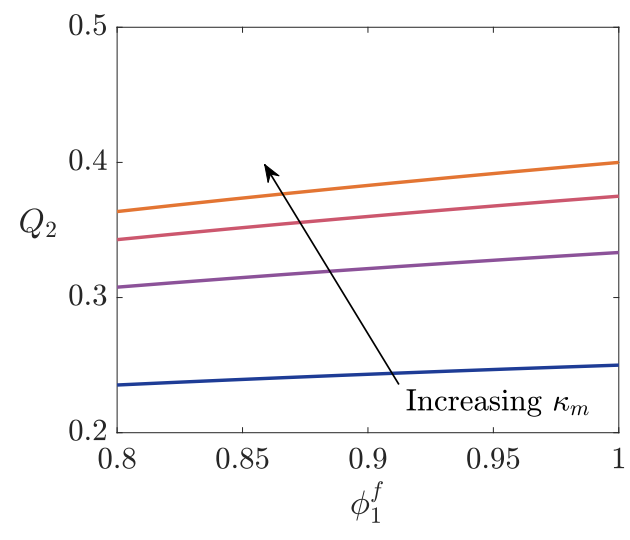

(b)

FIG. 9. Analytical inflow flux (B8a) and outflow flux (B8b) through a pipe with dimensionless pressure drop $\Delta p=1$ and porous membrane of permeance $\kappa_{m}=0.5,1,1.5,2$.

The flux profiles are shown in Fig. 9. Analogously to the results in Sec. III, the inflow flux decreases with increased fluid volume fraction [Fig. 9(a)]. For a decreased fluid volume fraction, there is a corresponding increased particle volume fraction. There is consequently less physical space available for the fluid to move, so for the same fixed pressure drop, the flow will speed up. On the downstream side of the membrane we observe that the outflow flux increases with the fluid volume fraction [Fig. 9(b)]. This shows that the total amount of fluid processed through the domain will increase when there are fewer particles in the system.

The results discussed in Sec. III and in this Appendix show how including particles in the system affects the flux through a system of two open domains separated by a porous membrane for a given pressure drop. Namely, we find that introducing particles speeds up the flow rate upstream of the membrane while decreasing the total amount of fluid processed by the system.

[1] R. D. Noble and S. A. Stern, Membrane Separations Technology: Principles and Applications (Elsevier, Amsterdam, 1995).

[2] K. M. Yao, M. T. Habibian, and R. O'Melia, Water and waste water filtration. concepts and applications, Environ. Sci. Technol. 5, 1105 (1971).

[3] R. van Reis and A. Zydney, Bioprocess membrane technology, J. Membr. Sci. 297, 16 (2007).

[4] Smart Separations Ltd, https://smartseparations.com/ (2020).

[5] V. E. Pereira, M. P. Dalwadi, E. Ruiz-Trejo, and I. M. Griffiths, Optimising the flow through a concertinaed filtration membrane, J. Fluid Mech. 913, A28 (2021).

[6] M. Wang, S. Mondal, and I. M. Griffiths, The role of fouling in optimizing direct-flow filtration module design, Chem. Eng. Sci. 163, 215 (2017).

[7] J. R. King and C. P. Please, Asymptotic analysis of the growth of cake layers in filters, IMA J. Appl. Math. 57, 1 (1996).

[8] P. Sanaei, G. W. Richardson, T. Witelski, and L. J. Cummings, Flow and fouling in a pleated membrane filter, J. Fluid Mech. 795, 36 (2016).

[9] Y. Sun, P. Sanaei, L. Kondic, and L. J. Cummings, Modeling and design optimization for pleated membrane filters, Phys. Rev. Fluids 5, 044306 (2020).

[10] G. S. Beavers and D. D. Joseph, Boundary conditions at a naturally permeable wall, J. Fluid Mech. 30, 197 (1967). 
[11] I. M. Griffiths, P. D. Howell, and R. J. Shipley, Control and optimization of solute transport in a thin porous tube, Phys. Fluids 25, 033101 (2013).

[12] I. M. Griffiths, A. Kumar, and P. S. Stewart, A combined network model for membrane fouling, J. Colloid Interface Sci. 432, 10 (2014).

[13] S. Mondal, I. M. Griffiths, and G. Z. Ramon, Forefronts in structure-performance models of separation membranes, J. Membr. Sci. 588, 117166 (2019). 